\title{
Object oriented simulation of Hybrid Renewable Energy systems focused on Supervisor Control
}

\author{
José María González de Durana \\ Universidad del País Vasco UPV/EHU \\ DISA Escuela Universitaria de Ingeniería \\ Nieves Cano 12, 01006 Vitoria \\ jtpgogaj@ehu.es
}

\author{
Oscar Barambones \\ Universidad del País Vasco UPV/EHU \\ DISA Escuela Universitaria de Ingeniería \\ Nieves Cano 12, 01006 Vitoria \\ oscar.barambones@ehu.es
}

\begin{abstract}
With eyes focused on simulation the authors review some of the main topics of Hybrid Renewable Energy Systems (HRES). Then they describe an Object Oriented model of a simple example of one of such systems, a micro-grid, oriented to designing a decentralized Supervisor Control. The model has been implemented using AnyLogic.
\end{abstract}

Keywords: Photo Voltaic (PV), Fuel Cell (FC), Hybrid Renewable Energy Systems (HRES), Distributed Energy Resources (DER), Renewable Energy Sources (RES), Agent Based Modeling \& Simulation (ABMS), Object Oriented Models (OOM), Hybrid Systems (HS), AnyLogic.

\section{Introduction}

Probably one of the biggest problem that humans have today is the excessive energy consumption from conventional sources, which results in the large climate change we are experiencing. In the author's opinion, the solution, if any, would go through a drastic reduction in energy consumption of each person in adition to a massive decentralization of production. It is not acceptable wasting energy for moving 1000 $\mathrm{kg}$ or more when our body is about $100 \mathrm{Kg}$, we are making a mess of consumption.

One can believe that in order to solve these problems it would be necessary to eliminate or drastically reduce all products and processes that pollute, such as automobile, airplane, electricity production from non-renewable sources, as well as associated activities like as freight transport and tourism. This would involve greater use of human and animal muscle power, and perhaps designing new manual appliances. It would be like turning the clock back. But it is clear that such ideas clash head on with the current economic models of market economy and globalization.

While not as dramatic, people are gradually ac- cepting some schemes aimed towards a solution to the problem, such as the tendency to decentralize the production, use of renewables energy sources, and manufacturing products such as automobiles with less energy consumption.

Recently, by a sort of special circumstances, some researchers focus their attention on Hybrid Renewable Energy Systems (HRES), and a number of stand alone, autonomous systems of this kind have been designed and built [3]. But HRES systems exhibit a complex structure and behavior, even in their simplest instances. While some new tools for structuring and seizing HRES systems appeared [9], traditional control design methods reveals to be insufficient, and other more adequate ones are appearing.

\section{Hybrid Renewable Energy Systems (HRES)}

More than $25 \%$ of the world's population still does not have electricity, and statistics said that at current rate the number will increase from 1.6 billions today to more than 2 billions in a 20 years horizon, so 100 million people instead of the current 40 millions need to be connected to an electrical supply each year during this period.

The vast majority of the non-electrified population are living in remote and rural areas of the developing countries. Of these, the majority will probably never be connected to an electric network, since such electric connections will remain, for a predictable future, anti-economic, as for the remoteness and related insurmountable geographical and physical obstacles.

As this is the case, electrical mini-grids or microgrids have proved to have a very high potential to participate actively in the electrification of remote and rural areas. Such grids may be fed by electricity from different renewable and conventional decentralized sources.

To build up micro-grids power electronic plays a key role and a new system design philosophy for hy- 


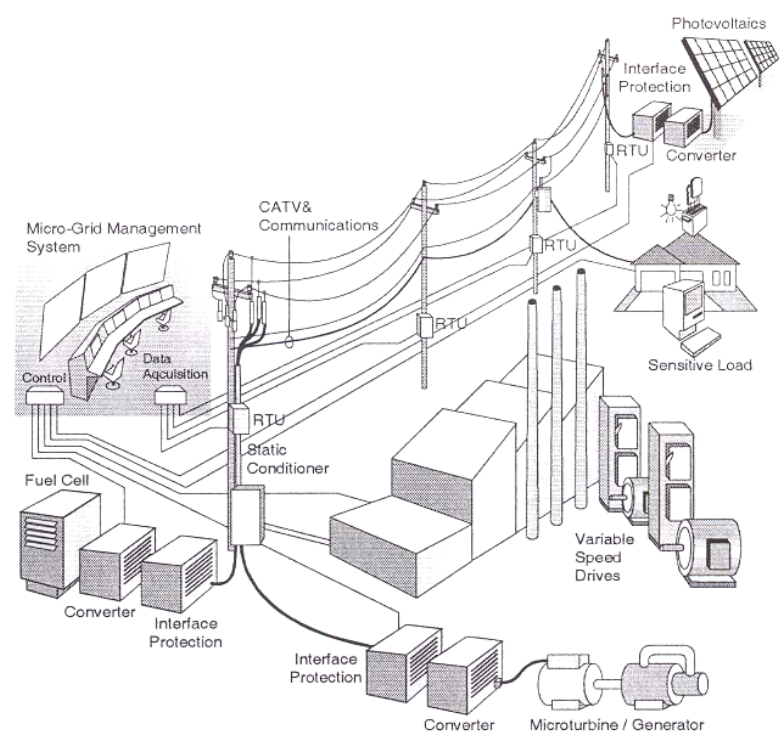

Figure 1. A microgrid drawing

brid power systems, where power electronic inverters are the corner stone, is coming.

Some interesting HRES applications are: mobile equipment, autonomous equipment, stand-alone PV energy systems, small village electricity power supply, water pumping and irrigation systems, communications power suply, lighting and small appliances, emergency power and lighting systems, stand-alone hybrid renewable energy systems, electric utility systems, mobile health emergency clinics, etc.

\subsection{Microgrids}

A micro-grid is formed by interconnection of small voltage generators, storage and controllable load distribution systems. Figure 1 shows a typical cartoon like micro-grid drawing. micro-grids can be connected to the main power network or be operated autonomously, similar to power systems of physical islands.

One of the main problems that a micro-grid fed by many decentralized energy sources faces is to manage the amount of active and reactive power to be produced by each power source in order to fulfill the load requirements. Such a problem, which is normally called load- or power-dispatch problem, is going to be discussed in this paper.

\subsection{Microgrid control}

One of the main problems that a micro-grid fed by many decentralized energy sources faces is to manage the amount of active and reactive power to be produced by each power source in order to fulfill the load requirements. Such a problem is normally called load-dispatch or power-dispatch problem.

For controlling a micro-grid seme strategies can be

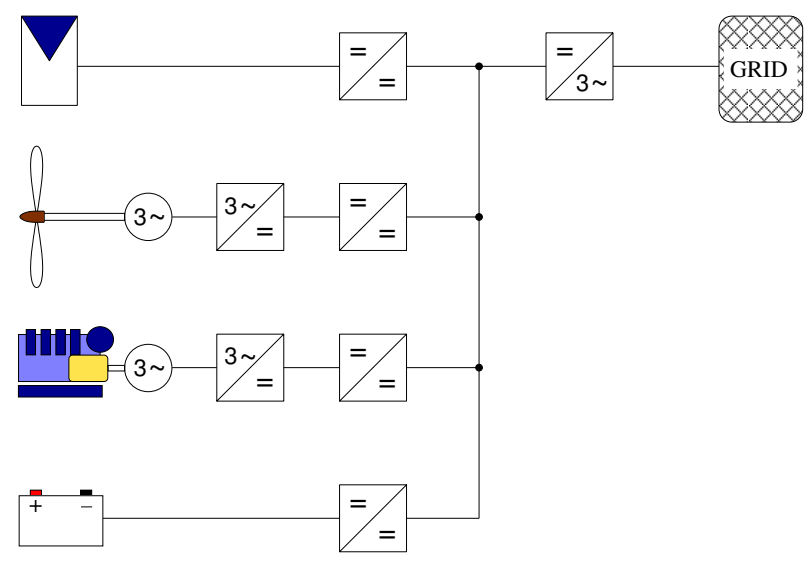

Figure 2. Microgrid configuration

used:

- Supervisor Control

- Local Decentralizad Control

- Centralized/decentralized Load Dispatching

Usually these strategeis can be combimed in applications which results in a number of combinations of possible to control types.

\subsection{Configuration}

In [5] the authors investigate an specific architecture of autonomous hybrid power system, based upon the utilization of a DC bus, a DC/AC inverter, and an individual DC/DC converter for each power source. In their opinion that is a good choice because, as they said, "the control/management units show ability to prioritize the functionality of the power supplies in such a way that the cheaper has the higher priority while guaranteeing the reliability of the whole system. A main advantage of the discussed system's architecture exists in providing a decoupling of the state values of the conversion systems from those of the grid through the use of the DC/DC converters". Figure 2 shows a sample of micro-grid configuration using this architecture.

By addition of DC/DC converters, the state values of the energy conversion sources become completely decoupled from each other and from the state values of the grid. The power production of the different sources becomes now freely controllable without affecting the state values of the grid.

Decoupling the state values means that the variations of the renewable resources like the velocity of the wind and the intensity of the solar radiation will not influence the state values of the electrical grid. These values are only controlled by the inverter. On the other hand, changes in the loads, which influence the state values of the grid will not affect the generation side. 


\section{Simulation}

Depending on simulation time or modeling horizon, three types of simulation are often distinguished:

- long term

- medium term

- short term

Modeling horizons are in some way related to the model abstraction degree: high abstraction level, middle abstraction level or low abstraction level [1].

In general a simulation model can be built in many different ways depending on the objectives of a particular problem to be solved.

Whenever a researcher like to simulate a system he satisfied with just a model on a particular facet, since the real system being extremely complex would not be possible to obtain a full model. It is therefore necessary to choose a viewpoint located sufficiently close as to give him a vision to perceive the features of the system behavior that are most important for our purposes.

From a close viewpoint the model is made using great detail and with a small time scale. Such models are used to model components with faster behavior, such as the transient behavior of electrical and electronic systems.

\subsection{Block based and object based simulation}

Block based (explicit causality, Simulink like) and Object Oriented (causality can be inferred, Dimola like) simulation languages are the two main categories to model HRES systems. In the authors opinion, object oriented languages are best positioned to cope with complexity that HRES systems exhibit.

An additional advantage of Object Oriented languages deals with the possibility of create Agent Based (AB) models. AB ranges from small, minimalist models to large-scale decision support systems.

\section{AnyLogic model}

AnyLogic is a simulation program developed by XjTek [10] using a subset of UML for Real Time (UML-RT) as a modeling language, and extended it to incorporate continuous behavior.

Three simulation paradigms are included in AnyLogic:

- System Dynamics (SD)

- Discrete Event (DE)

- Agent Based (AB)
SD and DE are traditional and $\mathrm{AB}$ is relatively new. This methods can be combined in a model, for example it is possible to include SD and DE methods into an Agent model, so each agent can be an hybrid system. With this kind of models a with a very high refinement level can be are obtained.

The Dynamic System (DS) paradigm, the main method used in block based programs like MatlabSimulink, is considered to be as a part of SD paradigm in AnyLogic since version 6 appeared.

Although AnyLogic is specially well featured to model Hybrid Systems, like electric and electronic circuits, there are not much papers about modeling electrical networks nor power system generation and distribution made using this software [8].

\subsection{Micro-grid simulation}

Hybrid Renewable Energy Systems include behaviors with very different values of time constants, so the establishment of a single model covering all details would have as advantage the universality, but it also have some drawbacks, as the difficulty to show in a clear mode the dominant behavior of the system and also, due the high complexity of the model, it would get an excessively long simulation time. The

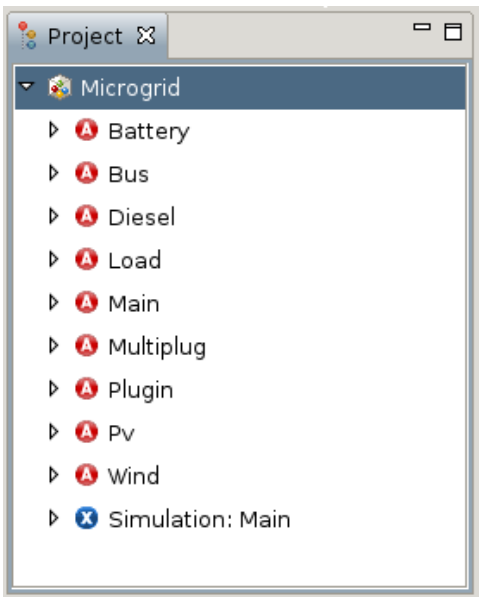

Figure 3. Project view

authors have developed a computational hybrid, object based, system modeling and simulation method, as a valuable tool for analysis, design and validation of micro-grids. Trying to simplify the exposition and the result analysis, and also for didactic reasons, the method is applied to a rather simple one.

The model has been built in AnyLogic using some formulae given in [3] for building some components. It is composed of 7 Active Object clases: Battery, Bus, Diesel, Load, Multiplug, $\mathrm{Pv}_{\mathrm{V}}$ and Wind besides the Main class. Figure 3 shows the AnyLogic Project window where you can the object tree. There is no room here to completely describe all this elements and therefore some esential aspects of them are described. 


\subsection{Wind system simulation}

Power output of wind turbine generator at a specific site depends on wind speed at hub height and speed characteristics of the turbine. Wind speed at hub height can be calculated by using power-law equation [3]:

$$
V_{\mathrm{z}}=V_{\mathrm{i}}\left(\frac{Z}{Z_{\mathrm{i}}}\right)^{x}
$$

where $V_{\mathrm{z}}$ and $V_{\mathrm{i}}$ are the wind speed at hub and reference height $Z$ and $Z_{\mathrm{i}}$, and $x$ is power-law exponent.

Power output $\mathrm{Pw}\left(\mathrm{kW} / \mathrm{m}^{2}\right)$ from wind turbine generator can be calculated as follows [3]:

$$
\begin{array}{ll}
P_{\mathrm{w}}=0, & V<V_{\mathrm{ci}} \\
P_{\mathrm{w}}=a V^{3}-b P_{\mathrm{r}}, & V_{\mathrm{ci}}<V<V_{\mathrm{r}} \\
P_{\mathrm{w}}=P_{\mathrm{r}}, & V_{\mathrm{r}}<V<V_{\mathrm{co}} \\
P_{\mathrm{w}}=0, & V>V_{\mathrm{co}}
\end{array}
$$

where $a=P_{r} /\left(V_{r}^{3}-V_{c i}^{3}\right), b=V_{c i}^{3} /\left(V_{r}^{3}-V_{c i}^{3}\right), P_{r}$ is

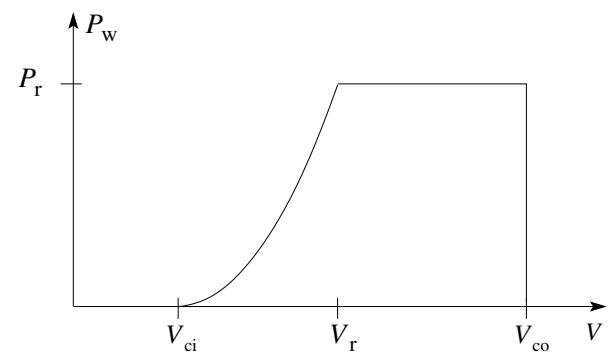

Figure 4. Power output

the rated power, $V_{c i}, V_{c o}$ and $V_{r}$ are the cut-in, cutout and rated speed of the wind turbine.

Actual power available from wind turbine is given by $[3]$ :

$$
P=P_{w} A_{w} \eta,
$$

where $A_{w}$ is the total swept area, $Z$ is efficiency of wind turbine generator and corresponding converters.

In [7] a time series model has been developed to simulate the real wind speed. Historical wind speeds can be obtained for some specific site, based on which, future hourly data are predicted using the time series model. The simulated wind speed $w_{s}$ is obtained from the mean wind speed $\mu_{t}$ and its standard deviation $\sigma_{t}$ at time $t$,

$$
w_{s t}=\mu_{t} \sigma_{t} y_{t}
$$

and the original data series set $y_{t}$ can be used to create a wind speed time series referred to as an ARMA (Auto-Regressive and Moving Average Model) stochastic process as

$$
\begin{aligned}
y_{t} & =\phi_{1} y_{t-1}+\phi_{2} y_{t-2}+\cdots+\phi_{n} y_{t-n} \\
& +\alpha_{t}-\theta_{1} \alpha_{t-1}-\theta_{2} \alpha_{t-2}-\cdots-\theta_{m} \alpha_{t-m}
\end{aligned}
$$

where $\phi_{i}, i=1,2, \ldots, n$ and $\theta_{j}, j=1,2, \ldots, m$, are the auto-regressive and moving average parameters of the model respectively, $\left\{\alpha_{t}\right\}$ is a normal white noise process with zero mean and variance of $\sigma_{a}^{2}$.

The above equation allows new values of $y_{t}$ to be calculated from current random white noise $\alpha_{t}$ and previous values of $y_{t-i}$. The hourly wind speeds incorporating the wind speed time series can be generated using this equation.

The ARMA model given in [3] (for specific site indicated in the paper) is

$$
\begin{aligned}
y_{t}= & 1.17 y_{t-1}+0.10 y_{t-2}-0.35 y_{t-3}+0.03 y_{t-4} \\
& +\alpha_{t}-0.50 \alpha_{t-1}-0.29 \alpha_{t-2}+0.13 \alpha_{t-3}
\end{aligned}
$$

where $\alpha_{t}$ is a white noise with zero mean and standard deviation $\sigma=5247602$.

An AnyLogic model of this process can be easily obtained by putting two Plain Variables from AnyLogic Palette to main window, naming them $a$ and $b$, giving them double double array type,

double[] a; double [] a;

and sizing and initializing them at model Start-up Code:

$\mathrm{y}=$ new double $[5] ; \mathrm{a}=$ new double [4];

$\mathrm{y}[0]=10 ; \mathrm{y}[1]=30 ; \mathrm{y}[2]=90 ; \mathrm{y}[3]=67 ; \mathrm{y}[4]=6.0$;

An hour is set as discrete time unit for ARMA process and so an event object from Palette and call it $\mathrm{h}_{-}$event is put in Main since it is able to produce hourly actions. Also a Clock object to show actual date and time during simulation run is placed in Main. The related Java code is:

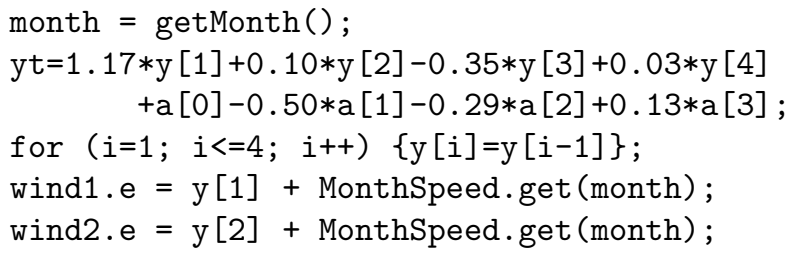

where wind1 and wind2 are two turbine generator objects described in the sequel.

\subsection{Photo Voltaic (PV) system simulation}

The solar radiation on an inclined plane is given by [3]:

$$
I_{T}=I_{b} R_{b}+I_{d} R_{d}+\left(I_{b}+I_{d}\right) R_{r}
$$

where $I_{b}$ and $I_{d}$ are direct normal and diffuse solar radiations, $R_{d}$ and $R_{r}$ are the tilt factors for the diffuse and reflected part of the solar radiations. $T_{T}$ depends on position of sun in the sky, which varies from month to month.

Hourly power output from PV system with an area $A_{p v}\left(\mathrm{~m}^{2}\right)$ on an average day of jth month, when total 
solar radiation of IT $\left(\mathrm{kW} \mathrm{h} / \mathrm{m}^{2}\right)$ is incident on $\mathrm{PV}$ surface, is given by [6]

$$
P_{s j}=I_{T_{j}} \eta A_{P V},
$$

where system efficiency $\eta$ is given by

$$
\eta=\eta_{m} \eta_{p c} P_{f}
$$

and the module efficiency $\eta_{m}$ is given by

$$
\eta_{m}=\eta_{r}\left[1-\beta\left(T_{c}-T_{r}\right)\right],
$$

where $Z_{r}$ is the module reference efficiency, $Z_{p c}$ is the power conditioning efficiency, $P_{f}$ is the packing factor, $\beta$ is the array efficiency temperature coefficient, $T_{r}$ is the reference temperature for the cell efficiency and $T_{c}$ is the monthly average cell temperature, calculated as

$$
T_{c}=T_{a}+\frac{\alpha \tau}{U_{L}} I_{T},
$$

where $T_{a}$ is the instantaneous ambient temperature, $U_{L} / \alpha \tau=I_{T, \mathrm{NOCT}} /\left(\mathrm{NOCT}-T_{a, \mathrm{NOCT}}\right)$, and $\mathrm{NOCT}$ is normal operating cell temperature, $T_{a, \mathrm{NOCT}}=20^{\circ} \mathrm{C}$ and $I_{\mathrm{T}, \mathrm{NOCT}}=800 \mathrm{~W} / \mathrm{m}^{2}$, for a wind speed of $1 \mathrm{~m} / \mathrm{s}$.

\subsection{Diesel engine}

If the load requirements are not met by either renewable energy system or by batteries (perhaps due to state of charge) then load requirements are met by operating diesel generator in HRES. The diesel generator shuld have a rated capacity at least equal to the maximum load and the current produced by the generator should not be greater than $C_{A h} / 5 \mathrm{~A}$, where $C_{A h}$ is the ampere hour capacity of the battery.

Overall efficiency of diesel generator is:

$$
\eta_{\text {overall }}=\eta_{\text {brakethermal }} \cdot \eta_{\text {generator }},
$$

where $\eta_{\text {brakethermal }}$ is brake thermal efficiency of diesel engine. Normally, diesel generators are modeled in the control of the hybrid power system in order to achieve required autonomy. It is observed that if the generator is operated at 70-90\% of full load then it is economical. In the absence of peak demand, diesel generators are normally used for meeting load requirements and for battery charging.

\subsection{Battery system}

Battery storage is sized to meet the load demand during non-availability period of renewable energy source, commonly referred to as days of autonomy. Normally days of autonomy is taken to be 2 or 3 days. Battery sizing depends on factors such as maximum depth of discharge, temperature correction, rated battery capacity and battery life. Required battery capacity in ampere hour is given by

$$
B_{\mathrm{rc}}=\frac{E_{\mathrm{c}(A h) D s}}{(D O D)_{\max } \eta_{t}}
$$

where $E_{\mathrm{c}(\mathrm{Ah})}$ is the load in ampere hour, $D_{\mathrm{s}}$ is the battery autonomy or storage days, $\mathrm{DOD}_{\max }$ is the maximum battery depth of discharge, $\eta_{t}$ is the temperature correction factor.

Difference between power generated and load decides whether battery is in charging or discharging state. The charge quantity of battery bank at the time $t$ can be calculated by

$$
E_{\mathrm{B}}(t)=E_{\mathrm{B}}(t-1)(1-\sigma)+\left(E_{\mathrm{GA}}(t)-E_{\mathrm{L}}(t) / \eta_{\text {inv }}\right) \eta_{\text {battery }}
$$

where $E_{\mathrm{B}}(t)$ and $E_{\mathrm{B}}(t-1)$ are the charge quantities of battery bank at the time $t$ and $t-1, \sigma$ is the hourly self-discharge rate, $E_{\mathrm{GA}}(t)$ is the total energy generated by renewable energy source after energy loss in controller, $E_{\mathrm{L}}(t)$ is load demand at the time $t, \eta_{\text {inv }}$ and $\eta_{\text {battery }}$ are the efficiency of inverter and charge efficiency of battery bank.

Charge quantity of battery bank is subject to the following constraints:

$$
E_{\mathrm{B}_{\mathrm{m}} \text { in }} \leq E_{\mathrm{B}}(t) \leq E_{\mathrm{B}_{\max }},
$$

where $E_{\mathrm{B}_{\max }}$ and $E_{\mathrm{B}_{\min }}$ are the maximum and minimum charge quantity of battery bank.

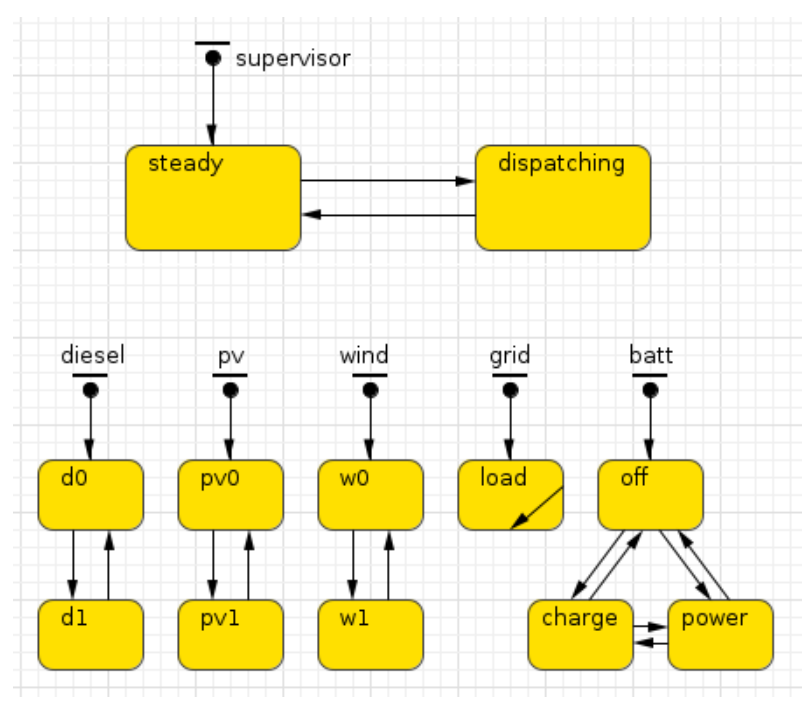

Figure 5. Supervisor control

\subsection{DC bus simulation in System Dynamics}

As already it is said, we will focus our simulation in the specific architecture for autonomous hybrid power system (HPS) investigated in [5] by their authors, based on utilization of a DC bus, a DC/AC inverter, and an individual DC/DC converter for each power source.

Using this scheme, a model of a micro-grid like as depicted in figure will be done in the following 2 .

The first modeling element is the DC bus (figure 2 ). Basically all we need is to write up the equations 
for the circuit in figure 6 , where the Thevenin equivalent circuit for each one is asumed $n$ electrical power sources, and that load is composed by $m$ switched and possible varying resistances.

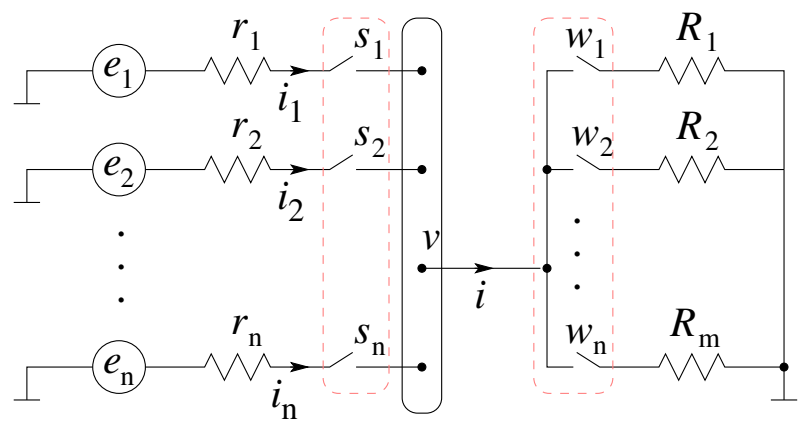

Figure 6. Basic connection circuit

The equivalent resistance to the $m$ load resistances connected in parallel is

$$
R=\frac{1}{\frac{w_{1}}{R_{1}}+\frac{w_{2}}{R_{2}}+\cdots+\frac{w_{m}}{R_{m}}}
$$

where $w_{i}=0$ if the switch is open and $w_{i}=1$ if the switch is closed, for $i=1, \ldots, m$. Then $v=R i$ and then the intensity flowing through generator line is

$$
i_{1}=\frac{e_{1}-v}{r_{1}}, i_{2}=\frac{e_{2}-v}{r_{2}}, \ldots, i_{n}=\frac{e_{n}-v}{r_{n}}
$$

if each switch is closed, or $i_{i}=0(i=1, \ldots, n)$, if the switch is open. So

$$
i=i_{1}+i_{2}+\ldots+i_{n}
$$

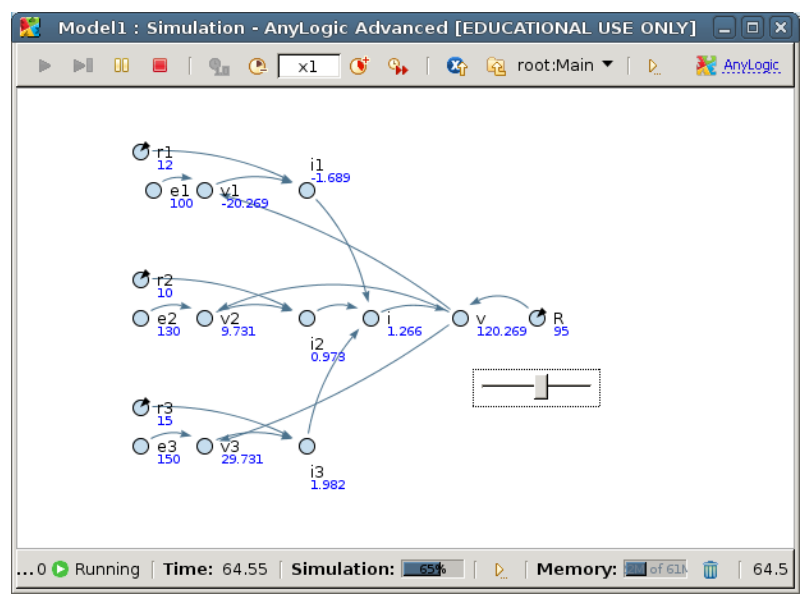

Figure 7. Basic Sistem Dynamics model

If one realize that there is a feedback from output voltage $v$ until each voltage source $e_{1}, e_{2}, \ldots, e_{n}$, it is not difficult to draw to the Forrester diagram that appears in figure 7 . The load resistance $R$ can be changed by the user during simulation by controlling the slider. This is the basic model to build all the other objects

\subsection{Supervisor Control}

As in [6], for designing supervisor control, the authors asume the main generation role is in charge of the wind subsystem while the solar subsystem would play a complementary role. However a rather different method to implement the control is proposed here: we use a centralized scheme for supervisor control and assume that load dispatching will be done in a decentralized manner (see figure 5).

Supervisor control performs a centralized operation. It is modeled with a statechart with two states: steady and dispatching. Transition from steady to dispatching is fired when it receives a message indicating that a change in load dispatchig must be done. Then the system changes to dispatchig state, performs the oportune change and returns to the steady state.

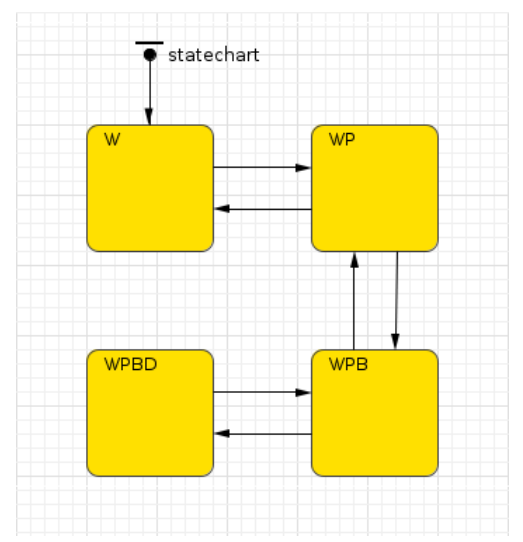

Figure 8. Supervisor

For supervisor control, as a first stage, a similar algorithm described in [6], but with four states implemented as statechart states in AnyLogic, is used. These states are:

State W (Wind). It represent conditions of sufficient wind power to fulfill the load demand without sun energy. In this state the wind subsystem supply total demand, the solar subsystem is no active and the battery bank is storing energy (recharge cycle). The system stay in this state until the total load demand of power exceeds the available wind power. When this event occurs the supervisor control switches to state WP, activating the solar module.

State WP. When load demand is greater than available wind power then transition from state $\mathrm{W}$ to state WP is fired. Wind subsystem is set for maximum generation and the solar subsystem is set to fulfill the load demand of power. 
State WPB. This mode of operation is characterized by both generation subsystems set to operate at their maximum energy conversion points. Moreover the battery bank is able to revert its energy flow, acting as power supplier instead as a recipient of energy. Operation in state WPB is maintained as long as the energy available in the battery bank is about a fixed percentage (said $24 \%$ ) of maximum stored energy, otherwise diesel generator is connected to fulfill the load demand.

\section{State WPBD.}

If sum of energy from wind, sun and battery are not sufficient to satisfy the load demand then an event occurs and transition from state WPB to WPBD is fired. When system enters this state, diesel generator is turned on to supply sufficient energy as to fulfill the load demand.
It can be shown that supply is guaranteed even if load demand of power or metheorological conditions change. This can be made specially illustrative and didactic by letting the user clicking at houses as order to change load demand of each one while historical meteorological conditions also change. In this way it is possible to study the microgrid behavior in all possible conditios which could be very interesting in order to sizing microgrid elements and designig its structure.

A Clock object (associated to Java calendar class) has been borrowed from AnyLogic Enterprise library to show data and time, from minutes and hours to months or even years.

Model also uses two AnyLogic TableFunction objects in order to store local meteorological historical data about wind velocity and sun radiation.

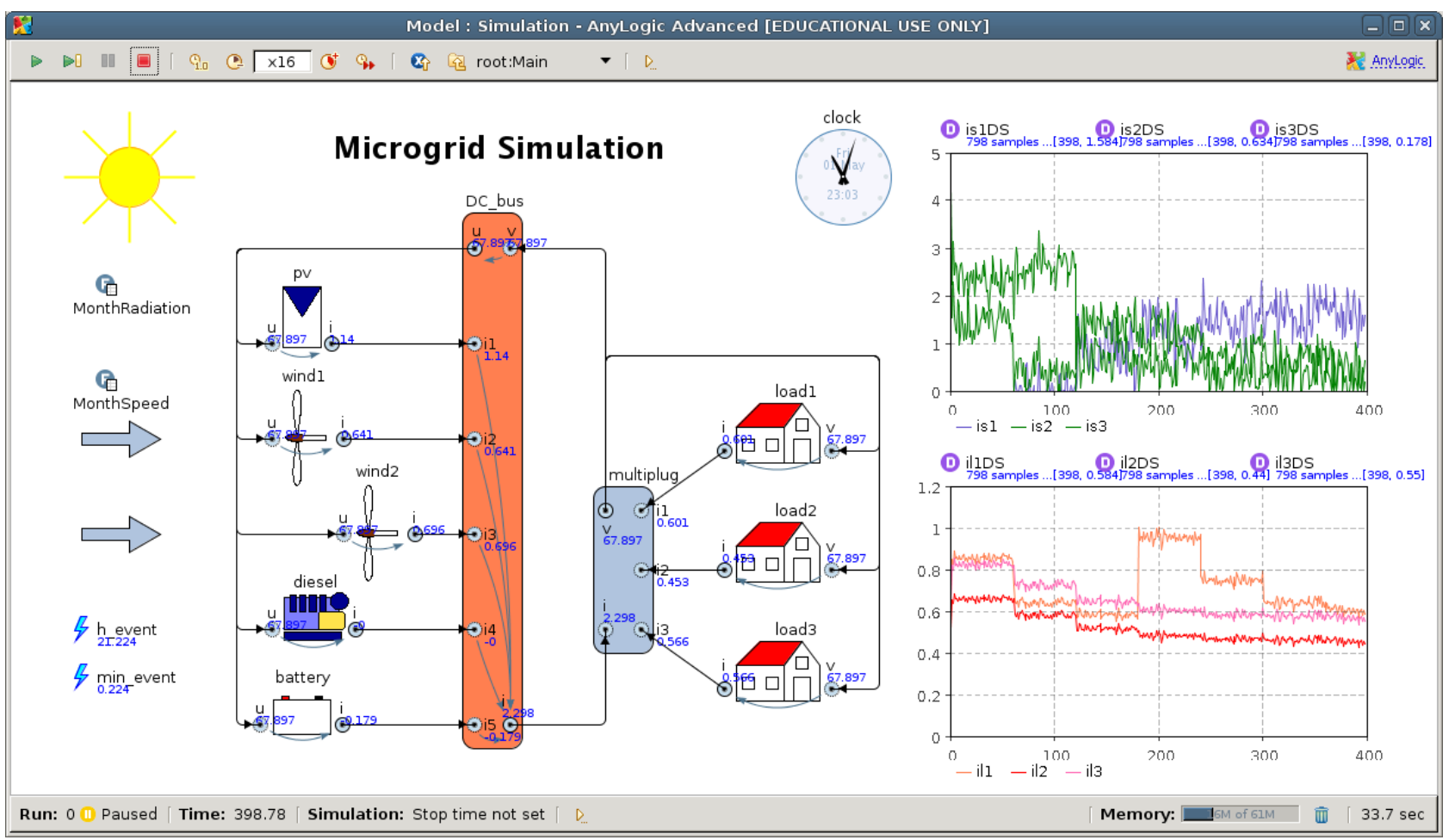

\subsection{Simulation results}

Once all the objects had been created it is possible to connect them and run simulation. Any number objects can be connected to DC_bus. Figure 4.7 represents the AnyLogic simulation window of a simple example with a photovoltaic generator (pv), two wind turbines (wind1 and wind2), a diesel generator (diesel) and a battery (battery) accumulator. Three loads (load1, load2 and load3) had been connected to multiplug (hiding a DC/AC converter) representing houses or any other kind of electric loads. Also source intensities $i_{s_{1}}, i_{s_{2}}, i_{s_{3}}$ and load intensities $i_{l_{1}}, i_{l_{2}}, i_{l_{3}}$ are represented.

\section{Conclusions}

An object oriented model for full simulation of micro-grids has been implemented using AnyLogic. The model is mainly intended to design and try micro-grids and it can be used as a tool for design, development and demonstration of control strategies specially Centralized Supervisor Control and Decentralized Load-Dispatch Control, design and demonstration of micro-grid operation strategies, design and trying of micro-grid communication buses, micro-grid ptimal design, and economic benefits demonstration.

The model can be used to analyze a number of 
topic design of micro-grids like as:

- Design, development and demonstration of control strategies specially centralized Supervisor control and decentralized load-dispatch control.

- Design and demonstration of micro-grid operation strategies.

- Design and trying of micro-grid communication buses.

- Optimal micro-grid design

- Economic benefits demonstration.

\section{Acknowledgments}

The authors would like to express their appretiation to Sergey Suslov Victor Gleim, Pavel Lebedev, Sergey Suslov, Stanislav Fedotov, Andrei Borshchev, Alexei Filippov, etc. from XJ Technologies Company, for their valuable explanations about AnyLogic topics and their unpayable help answering all the questions they asked.

They are also very grateful to the Basque Government by the support of this work through the project S-PE07UN04 and to the UPV/EHU by its support through project GUI07/08.

\section{References}

[1] A. Borshchev and A. Filippov. From System Dynamics and Discrete Event to Practical Agent Based Modeling: Reasons, Techniques, Tools. The 22nd International Conference of the System Dynamics Society, July 25 - 29, 2004, Oxford, England.

[2] Sonia Leva, Dario Zaninelli. Hybrid renewable energy-fuel cell system: Design and performance evaluation. Electric Power Systems Research 79 (2009) 316-324. Elsevier.
[3] M.K. Deshmukh, S.S. Deshmukh. Modeling of hybrid renewable energy systems. Renewable and Sustainable Energy Reviews 12 (2008) $235-249$

[4] Egon Ortjohann, Osama Omari, Md. Muzibur Rahman1and Danny Morton, Active \& reactive power dispatch in isolated mini-grids fed by decentralized power sources. 3rd International Conference on Electrical \& Computer Engineering ICECE 2004, 28-30 December 2004, Dhaka, Bangladesh.

[5] E. Ortjohann, O. Omari, R. Saiju, N. Hamsic, D. Morton, A Simulation Model For Expandable Hybrid Power Systems. University of Applied Sciences Südwestfalen, Division Soest, Laboratory of Power Systems and Power Economics. Internal Report.

[6] F. Valenciaga, P.F. PulestonSupervisor Control for a Stand-Alone Hybrid Generation System Using Wind and Photovoltaic Energy. IEEE Transactions on Energy Conversion, VOL. 20, NO. 2, JUNE 2005.

[7] R. Billinton, H. Chen and R. Ghajar, Time-series Models for Reliability Evaluation of Power Systems Including Wind Energy, Microelectron. Reliability, Vol.36. No.9. 1996, pp 1253-1261.

[8] Y.G. Karpov, R.I. Ivanovski, N.I. Voropai, D.B. Popov, "Hierarchical Modeling of Electric Power System Expansion by AnyLogic Simulation Software", Power Tech, 2005 IEEE Russia, 27-30 June 2005 Page(s):1 - 5

[9] www.nrel.gov/homer/

[10] XJ Technologies, St Petersburg, Russia. http://www.xjtek.com 